\title{
CAPTEUR ULTRASONORE HAUTE-FREQUENCE À ABSORBEUR ACTIF
}

\author{
M. OURAK, M. RAVEZ, B. NONGAILLARD et M. OUAFTOUH \\ Laboratoire d'Opto-Acousto-Electronique (URA 832 CNRS), Université de Valenciennes, ENSIMEV, \\ BP. 311, F-59304 Valenciennes, France
}

\begin{abstract}
Résumé : L'utilisation des sondes ultrasonores haute fréquence (100MHz et plus) dans le domaine biomédical, permet la caractérisation et la visualisation de structures de plus en plus fines. Les capteurs haute fréquence habituellement utilisés sont constitués d'un transducteur piézoélectrique de faible épaisseur collé sur une ligne à retard. Compte tenu de la gamme de fréquence utilisée, en plus des problèmes d'atténuation et d'adaptation, les multiples réflexions dans la ligne à retard introduisent un bruit ultrasonore qui entraîne une diminution de la sensibilité du capteur. Pour pallier à ces inconvénients, nous avons réalisé une sonde ultrasonore $(100 \mathrm{MHz})$ sans ligne à retard émettant directement dans le milieu biologique. Pour augmenter la bande passante, donc la résolution axiale du capteur, nous avons réalisé un absorbeur actif constitué d'un autre élément piézoélectrique. Dans notre article, nous présentons une comparaison entre résultats théoriques et expérimentaux obtenus avec ce nouveau capteur.

Abstract : The characterization and visualization of smaller and smaller structures become nowadays feasible in the biomedical ultrasonics area, owing to the design and construction of very high frequency ultrasonic probes ( above $100 \mathrm{MHz}$ ). These probes consist in a very thin piezoelectric transducer cemented on to an a delay line. The classical problems of ultrasonic attenuation and electrical and mechanical impedance matching are encountered. Moreover, in the used frequency range, multiple reflections on the various boundaries induce an acoustic noise which reduces the probe sensitivity. To overcome this, an ultrasonic probe radiating directly into biological medium, without buffer delay line has been built. An active damping using a second piezoelectic transducer has been designed in order to increase the available frequency bandwidth and then the axial resolution of the probe. In this paper, the experimental and theoritical results for this new kind probe are computed.
\end{abstract}

\section{INTRODUCTION}

Il est bien connu que l'amortissement arrière ainsi que l'adaptation mécanique par la face avant élargissent la bande passante d'un transducteur piézoélectrique. En plus, l'adaptation électrique du transducteur à l'impédance interne du générateur contribue à l'amélioration de la bande passante [1-4].

Dans une récente publication [5], nous avons montré qu'il était possible de simuler lamortisseur arrière à l'aide d'un transducteur chargé électriquement de manière à diminuer le volume et le poids de l'amortisseur arrière en basses fréquences.

Dans cet article, nous présentons la réalisation d'un nouveau capteur ultrasonore à $100 \mathrm{Mhz}$ utilisant un absorbeur actif. L'utilisation de transducteurs haute fréquence (très faible épaisseur) nécessite l'emploi d'un support. Nous comparerons les différents résultats obtenus avec ce capteur à ceux donnés par un transducteur classique sans amortisseur actif. 


\section{I - ANALYSE DE LA METHODE - DESCRIPTION DU CAPTEUR HAUTE FREQUENCE}

Dans la méthode proposée, le capteur (fig.1) est constitué d'un transducteur émetteur $\mathrm{T}_{1}$ collé sur un autre transducteur $T_{2}$ chargé par une impédance électrique $Z_{0}$. Le transducteur $T_{2}$ est lui-même collé sur un support en corindon d'impédance acoustique $Z_{s}=4310^{6} \mathrm{Kg} \cdot \mathrm{m}^{-2}$. $\mathrm{s}^{-1}$. L'ensemble formé par $T_{2}$ et son support en corindon constitue l'amortisseur arrière dont l'impédance mécanique complexe $\mathrm{Z}_{2}=\mathrm{R}_{2}+\mathrm{j} \mathrm{X}_{2}$ dite "impédance ramenée", dépend de la fréquence d'excitation.

La partíe $M$ permet une adaptation électrique du transducteur $T_{1}$ au générateur $(R g=50 \Omega)$. L'impédance $Z_{0}=R_{0}+j X_{0}$ représente la charge du transducteur $T_{2}$.

Ajoutons que le matériau constituant $\mathrm{T}_{1}$ et $\mathrm{T}_{2}$ est du Niobate de Lithium $\left(\mathrm{LiNbO}_{3}\right)$ d'impédance mécanique $Z=3510^{6} \mathrm{Kg} \cdot \mathrm{m}^{-2} \cdot \mathrm{s}^{-1}$. L'épaisseur commune $(e=37 \mu \mathrm{m})$ est choisie pour assurer une fréquence de résonance $\mathrm{F}_{0}$ de $100 \mathrm{MHz}$.

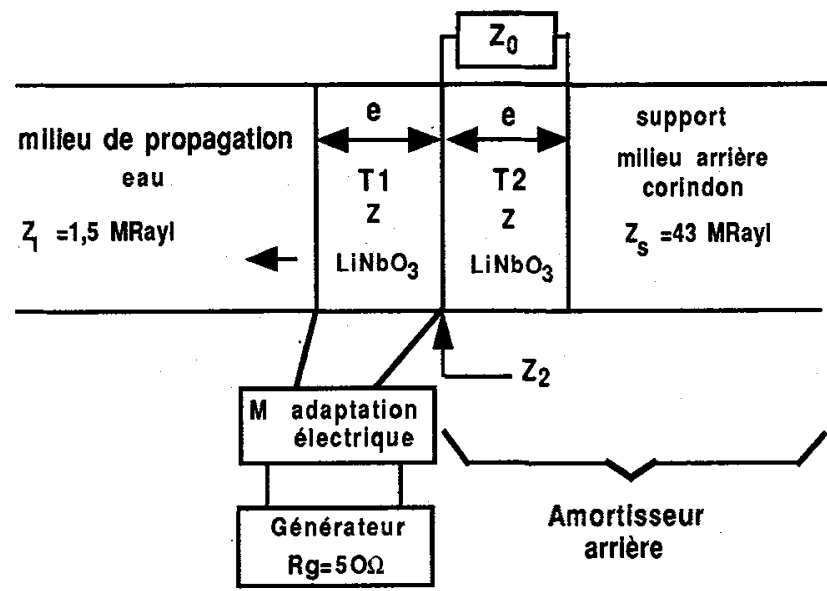

Fig.1: Shéma du capteur avec absorbeur actif .

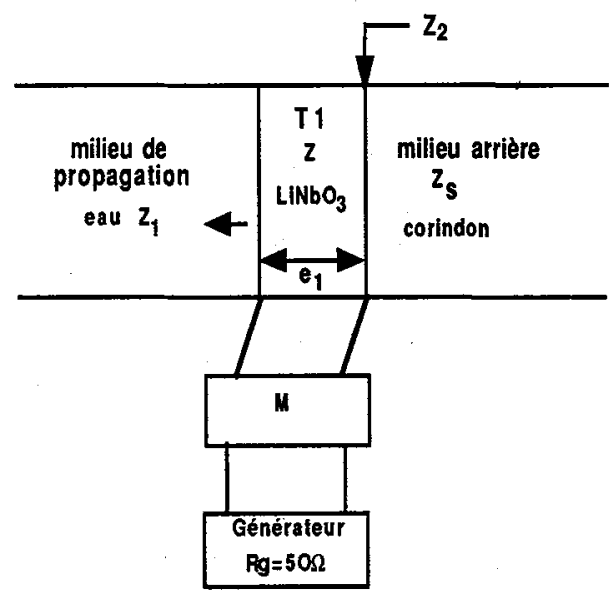

Fig.2: capteur sans absorbeur actif .

\section{II - PUISSANCE ACOUSTIQUE ET BANDE PASSANTE}

Pour mettre en évidence l'amélioration apportée par ce nouveau type de capteur, nous comparerons les deux configurations correspondant aux figures 1 et 2 .

La puissance acoustique $\mathrm{P} 1$ dans le milieu de propagation d'impédance mécanique réelle $\mathrm{Z}_{1}$ est donnée par la relation:

$$
P_{1}=\frac{p^{2}|1|^{2}}{\omega^{2} \varepsilon^{2} A} \operatorname{Re}\left(Z_{1}\right)\left|\frac{Z(\cos \theta-1)+j Z_{2} \sin \theta}{Z\left(Z_{1}+Z_{2}\right) \cos \theta+j\left(Z^{2}+Z_{1} Z_{2}\right) \sin \theta}\right|^{2}
$$

où Re signifie "partie réelle de", $\varepsilon$ la permittivité électrique, $\mathrm{p}$ le module piézoélectrique et $\theta=\pi \mathrm{F} / \mathrm{F}_{0}$, A la surface active du transducteur et I le courant électrique traversant le transducteur $T_{1}$.

Les équations de base de la piézoélectricité [6] nous permettent d'établir la relation donnant l'impédance électrique $Z_{\text {él }}$ :

$$
Z_{\dot{e} l}=-\frac{j e}{\omega \varepsilon A}+\frac{p^{2}}{\omega^{2} \varepsilon^{2} A}\left[\frac{2 Z(\cos \theta-1)+j\left(Z_{1}+Z_{2}\right) \sin \theta}{Z\left(Z_{1}+Z_{2}\right) \cos \theta+j\left(Z^{2}+Z_{1} Z_{2}\right) \sin \theta}\right]
$$

De même, nous avons déterminé l'impédance ramenée $Z_{2}$ : 


$$
Z_{2}=Z \frac{\left[\frac{p^{2}}{\omega^{2} \varepsilon^{2} A}(\cos \theta-1)+\left(Z_{0}-j \frac{e}{\omega \varepsilon A}\right)\left(Z_{1} \cos \theta+j Z_{s} \cdot \sin \theta\right)+\frac{p^{2}}{\omega^{2} \varepsilon^{2} A Z}\left(Z(\cos \theta-1)+j Z_{1} \sin \theta\right)\right]}{\left(Z_{0}-j \frac{e}{\omega \varepsilon A}\right)\left(Z \cos \theta+j Z_{1} \sin \theta\right)+j \frac{p^{2}}{\omega^{2} \varepsilon^{2} A} \sin \theta}
$$

\section{Résultats théoriques :}

Les figures 3.a et 3.b donnent respectivement l'évolution de la puissance acoustique en fonction de la fréquence et la réponse impulsionnelle du transducteur dans le milieu de propagation dans le cas d'un seul transducteur (absence de l'absorbeur actif). Les figures 4.a et 4.b représentent l'évolution des mêmes grandeurs dans le cas de la figure 2 (présence de l'absorbeur actif).

Nous constatons un élargissement de la bande passante (fig.4.a) ainsi qu'un amortissement du signal acoustique. Cependant, l'élargissement de la bande passante n'est pas très important. Ceci est dû à la forte impédance mécanique du support (proche de celle du $\mathrm{LiNbO}_{3}$ ), l'adaptation mécanique est alors pratiquement réalisée.

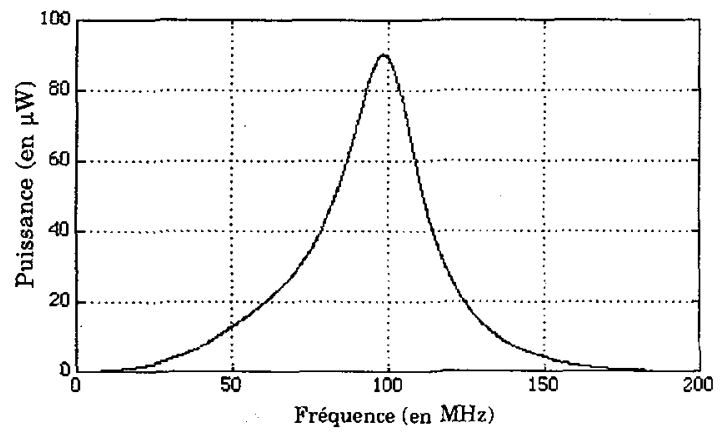

Fig.3.a: Puissance acoustique sans $\mathrm{T}_{2}$

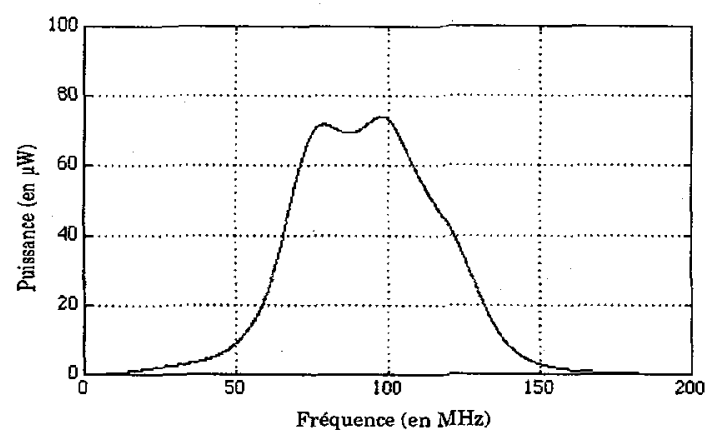

Fig.4.a: Puissance acoustique avec $\mathrm{T}_{2}$

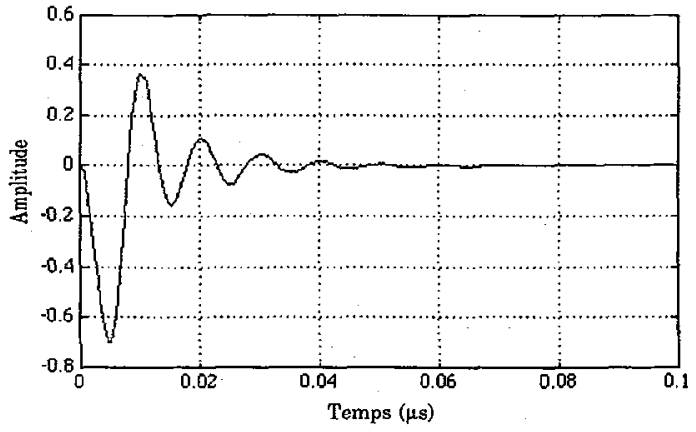

Fig.3.b: Diagramme temporel sans $\mathrm{T}_{2}$

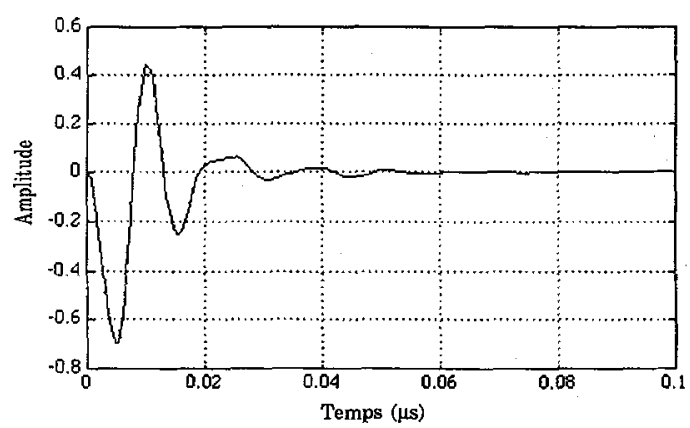

Fig.4.b: Diagramme temporel avec $T_{2}$

\section{Résultats expérimentaux :}

Compte tenu du choix porté sur le matériau constituant le support de $\mathrm{T}_{2}$ et pour des raisons techniques, nous avons préféré, dans un premier temps, analyser l'efficacité de l'absorbeur actif. Pour ce faire nous avons étudié le signal réfléchi par la face arrière du support (fig.5). Les figures 6.a et 6.b donnent respectivement:

- le diagramme temporel dans le cas où le transducteur $T_{2}$ n'est pas chargé par l'impédance $Z_{0}$

- le diagramme temporel dans le cas où le transducteur $T_{2}$ est chargé par l'impédance $Z_{0}$ 


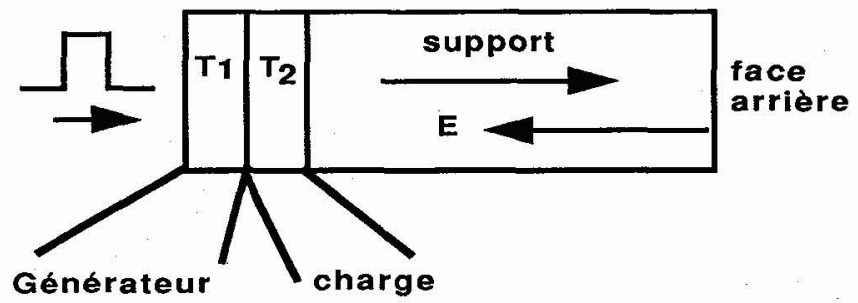

Fig. 5 : Analyse du signal réfléchi par la face arrière du support

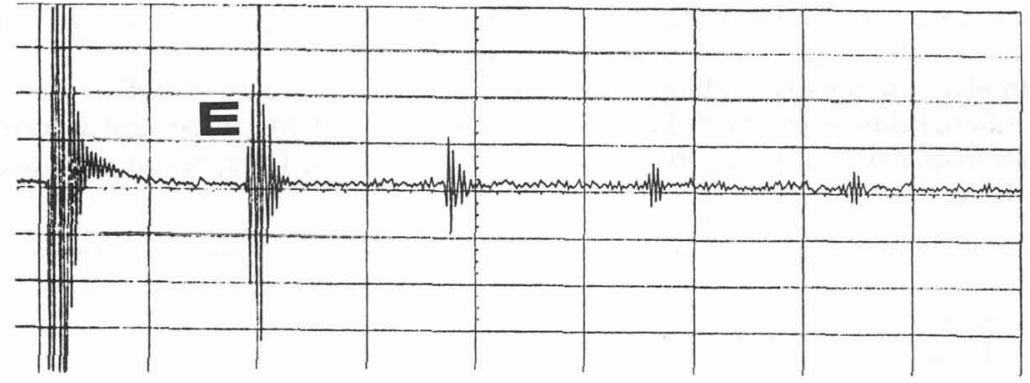

Fig.6.a:

Diagramme temporel avec $\mathrm{T}_{2}$ non chargé

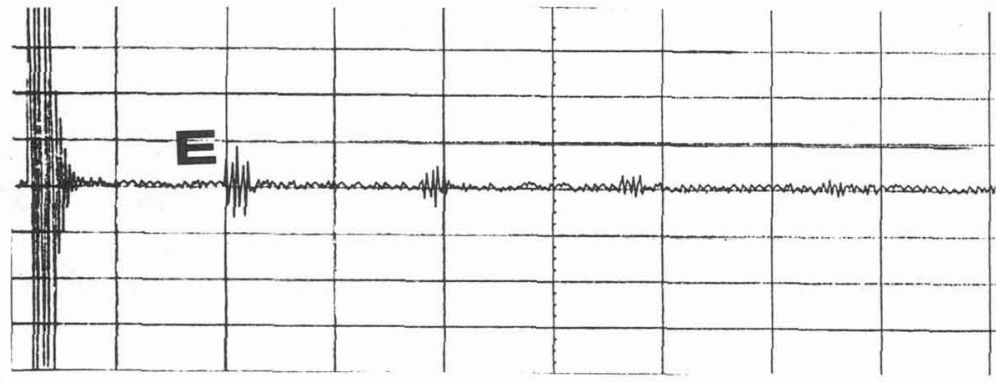

Fig.6.b:

Diagramme temporel avec $T_{2}$ chargé (forte atténuation du signal réfléchi par la face arrière.

horiz. : $1 \mu \mathrm{s} /$ div

vert. : $50 \mathrm{mV} / \mathrm{div}$

\section{III - CONCLUSION}

Cette étude montre que la présence du transducteur $\mathrm{T}_{2}$ quand il est bien adapté, peut constituer un absorbeur actif très efficace. Ces résultats sont très encourageants et nous permettent d'envisager dans un proche avenir, la conception de sondes haute fréquence dépourvues de bruit acoustique causé par les réflexions multiples au niveau de la ligne à retard.

Le domaine biomédical, très exigeant quant à la qualité de la résolution axiale trouvera là un outil très performant.

\section{REFERENCES}

[1]- J. Souquet, P. DeFraould, and J. Desbois, "Design of Low-Loss Wideband Ultrasonic Transdu cers for Noninvasive Medical Applications,"IEEE Trans.Sonics Ultrason.26 (2),75-81 (1979).

[2]- J. H. Goll,"The Design of Broad-Band F luid-Loaded Ultrasonic Transducers,"IEEE Trans. Sonics Ultrason. $26(6), 385-393$ (1979).

[3]- R. H. Coursant, "Les transducteurs ultrasonores", Rev. Electron. Phys. Appl. 22,129-141 (1979).

[4]- S. H. Van Kervel and J. M. Thijssen, "A Calculation Scheme for the Optimum Design of Ultrasonic Transducesr", Ultrasonics 21, 134-140 (1983).

[5]- M. G. Gazalet, M. Houzé, P.Logette, C.Bruneel, and R.Torguet, "Dummy load backing of ultrasonic transducers for bandwidth enhancement", J. Acoust. Soc. Am.83(3),1180-1182 March1988.

[6]- E.Dieulesaint, D.Royer, Ondes élastiques dans les solides, Ed.Masson (1974). 